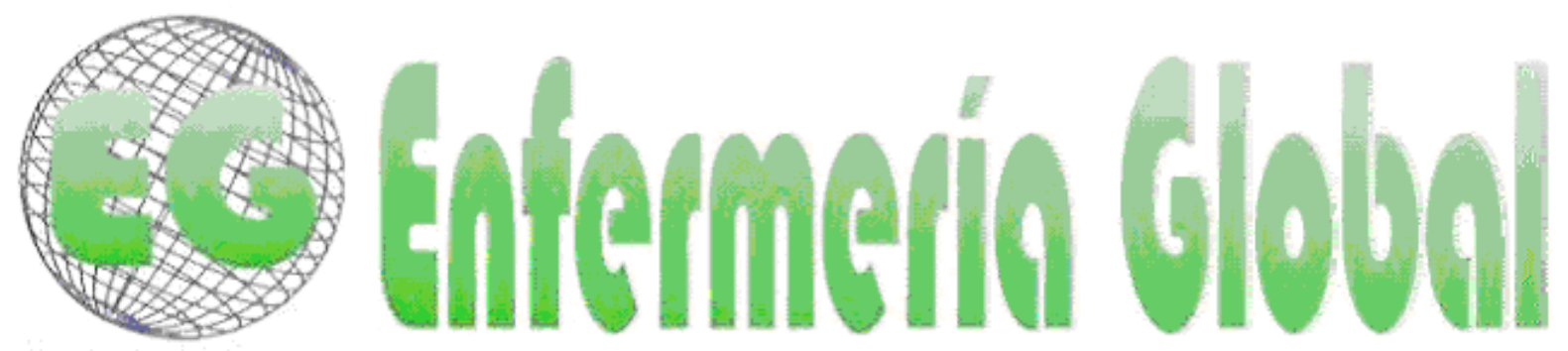

\title{
Experiencias en un grupo de apoyo para madres con hijos menores de un año
}

Experiences in a support group for mothers with children under a year

\section{*Berlanga Fernández, Sofía ${ }^{* *}$ Pérez Cañaveras, Rosa $\mathrm{M}^{\mathrm{a}}{ }^{* *}$ Vizcaya Moreno, $M^{a}$ Flores *Berlanga Fernández, Francisco *González López, Noelia Antonia}

\author{
*Enfermera. Instituto Catalán de la Salud. E-mail: sbf10@hotmail.es "**Enfermera. Universidad de \\ Alicante. \\ Palabras clave: Modelos; Mercer; Beck; Etnografía; Cualitativo, Observación Participante; Grupos de \\ Apoyo. \\ Keywords: Mercer; Beck; Etnography; Qualitative, Participant observation; support groups
}

\section{RESUMEN}

El proceso de la maternidad, según Mercer, implica que la madre nazca psicológicamente, dando lugar a una nueva identidad en constante crecimiento y desarrollo. El modelo de esta autora puede servir a la enfermera para valorar, planificar, ejecutar y evaluar el cuidado enfermero de las madres y sus bebés en sus intervenciones

Este estudio cualitativo, de enfoque etnográfico, dirigido a grupos de madres con hijos menores de un año, utilizará la observación participante y el diario de campo para describir y relacionar los contenidos de las dinámicas grupales con el desarrollo del rol maternal. Se identifican 3 categorías y 50 códigos y se destaca que las enfermeras, como profesionales competentes en aspectos conceptuales y técnicos propios de su disciplina, utilizan los modelos de Mercer y Beck para acompañar a las madres coordinándose con otros profesionales.

\section{ABSTRACT}

The process of motherhood, according to Mercer, implies the mother's psychological birth, creating a new identity under constant growth and development. The model of this author can serve to the nurse to value, plan, implement and assess the nurse care of mothers and their babies in their interventions.

This is a qualitative study, of an ethnographic approach, directed to groups of mothers with children under a year that would use the participant observation and the field diary to describe and relate the contents and the group dynamics with the development of the maternal role. Three categories and 50 codes are identified and it is enhanced that nurses, as competent professionals in conceptual and 
technical aspects of their discipline, use the Mercer's and Beck's models to accompany the mothers and coordinating with other professionals.

\section{INTRODUCCIÓN}

El proceso de la maternidad implica que la madre nazca psicológicamente al igual que su bebé nace físicamente, dando lugar en su mente a una nueva identidad: el sentido de ser madre ${ }^{(1)}$.

El establecimiento de la identidad de una madre en el nacimiento contribuye al desarrollo piscosocial de la mujer ${ }^{(2)}$.En este proceso, las madres adquieren una actitud mental que organiza sus vidas mentales, reorientando sus preferencias, placeres y reestructurando algunos de sus valores personales. Esta actitud se mantendrá durante toda la vida, aunque no ocupe el primer lugar, siempre estará presente, a la espera y emergerá cuando sea necesario. De esta manera se afirma que el nacimiento de una madre está en constante crecimiento y desarrollo ${ }^{(1)}$.

En esta línea, Ramona Mercer propone que el término adopción del rol maternal se sustituya por convertirse en madre ${ }^{(2)}$, considerándolo como un proceso de evolución continua e infinita, influido por factores relacionados con el entorno, el niño y la madre y configurado por distintas etapas en el primer año de vida: compromiso y preparación; conocimiento, práctica y recuperación física y normalización e integración de la identidad maternal ${ }^{(2)}$.

Acompañando a la madre en este proceso, está la enfermera que puede servirse del modelo de Mercer para proporcionar cuidados enfermeros a las nuevas madres y a sus bebés en las intervenciones individuales y/o grupales que realice ${ }^{(3)}$.

A través de las dinámicas grupales, la enfermera ofrecerá información y conocimientos a la vez que dinamizará a un conjunto de participantes permitiendo aprender de sus aportaciones, generar emociones, intervenir sobre actitudes, facilitar el aprendizaje por imitación y proporcionar seguridad al individuo ${ }^{(4)}$. Además, la evidencia sugiere que intervenciones intensivas en el primer año del postparto pueden producir una diferencia significativa tanto en los resultados maternos como en los del lactante ${ }^{(5)}$.

En el centro de Atención Primaria de Salud, Amadeo Torner, se vienen realizando desde hace nueve años, dinámicas grupales a madres con sus hijos menores de un año, conducidas por una psicóloga clínica y una enfermera, que acompañan a las familias en su proceso de maternidad.

Sirviéndonos de este método de Educación para la Salud, se pretende realizar un estudio con los objetivos de: describir los contenidos de las dinámicas grupales; relacionar los contenidos trabajados en el grupo con el desarrollo del rol maternal y aportar la teoría enfermera en este tipo de actividad, debido, entre otras cosas, a que a pesar de los esfuerzos continuados de investigación para identificar las variables relacionadas con la transición a la maternidad, los profesionales de la salud han hecho pocos progresos en traducir el conocimiento de estas variables en la práctica ${ }^{(5)}$. 


\section{MATERIAL Y MÉTODOS}

Estudio con diseño cualitativo y enfoque etnográfico. La técnica utilizada es la observación participante, en la que la investigadora principal se incluyó en un grupo de madres con sus hijos menores de un año en el transcurso de siete sesiones de carácter semanal en el Centro de Atención Primaria de Salud (CAP) Amadeo Torner en Hospitalet del Llobregat, entre abril y mayo del 2012.

En cada sesión, de dos horas y media de duración, participaron diez madres y sus bebés, las cuales fueron elegidas por sus enfermeras de referencia. Para ser incluidas en el grupo, las madres debían comprometerse en asistir a las sesiones y no podían presentar dificultad idiomática en la comunicación

La enfermera, como investigadora principal, participaba en todas las sesiones del grupo, utilizando el diario de campo como instrumento en la recogida de datos.

El diario de campo ha sido minuciosamente analizado, dividiendo los textos del diario en unidades de significado englobadas en diferentes categorías atendiendo a los factores que Ramona Mercer describe en su Teoría del rol maternal-convertirse en madre ${ }^{(3)}$, a los conceptos que Beck describe en su Teoría de la depresión postparto ${ }^{(3)}$ y a conceptos de promoción de la salud y prevención de la enfermedad. A partir de estas categorías se obtuvieron las codificaciones pertinentes.

Tras el análisis del diario de campo y con el objetivo de ofrecer rigor a la información obtenida, tanto las categorizaciones y codificaciones, como los resultados, fueron discutidos y analizados con la psicóloga que participó en el taller. Ante diferencias de interpretación, se consultó a otras enfermeras participantes en dinámicas grupales similares con el objetivo de resolverlas.

Finalmente, debe comentarse que se respetaron los criterios éticos que el comité de ética de nuestra institución solicita. Se pidió conformidad a las madres para poder tomar notas de campo y en todo el proceso los datos fueron registrados y analizados sin comprometer la identidad de las participantes.

\section{RESULTADOS}

Se han identificado un total de 3 categorías: "características de Mercer", "Conceptos de Beck" y "Promoción de la salud y prevención de la enfermedad" (tabla 1).

Tabla 1: Frecuencia de las categorías identificadas según los autores de referencia (3)

\begin{tabular}{lcc}
\hline Categorías & Frecuencia absoluta & Frecuencia relativa \\
\hline Factores de Mercer & 753 & $63,8 \%$ \\
Conceptos de Beck & 85 & $7,2 \%$ \\
$\begin{array}{l}\text { Promoción de la salud y } \\
\text { prevención de la enfermedad }\end{array}$ & 343 & $29 \%$ \\
Total & 1180 & $100 \%$ \\
\hline
\end{tabular}

Estas categorías incluyen un total de 50 códigos, ordenados en función del número de veces en que aparecen (frecuencia absoluta). Éstas son:

La primera categoría, “ características de Mercer" (tabla 2), incluye los factores: apoyo social, señales de los lactantes, unión, tensión debida al rol , características del niño, familia, compañero, adopción del rol materna, relación madre-padre, autoconcepto, 
funcionamiento de la familia, ansiedad, estrés, gratificación-satisfacción, temperamento del niño, identidad materna, actitudes respecto a la crianza, flexibilidad, autoestima y percepción de la experiencia del nacimiento.

\begin{tabular}{lcc} 
Tabla 2: Frecuencias de los ítems relacionados con las características definidas por Mercer (3) \\
\hline Ítems Factores de Mercer & Frecuencia absoluta & Frecuencia relativa \\
\hline Apoyo social & 240 & $32 \%$ \\
Señales de los lactantes & 129 & $17,2 \%$ \\
\hline Unión & 53 & $7 \%$ \\
Tensión debida al rol & 44 & $5,9 \%$ \\
Características del niño & 42 & $5,5 \%$ \\
\hline Familia & 33 & $4,4 \%$ \\
Compañero & 32 & $4,2 \%$ \\
Adopción del rol maternal & 29 & $3,9 \%$ \\
Relación madre-padre & 27 & $3,6 \%$ \\
\hline Autoconcepto & 20 & $2,7 \%$ \\
Funcionamiento de la familia & 18 & $2,4 \%$ \\
\hline Ansiedad & 17 & $2,2 \%$ \\
\hline Estrés & 13 & $1,8 \%$ \\
Gratificación- satisfacción & 12 & $1,6 \%$ \\
Temperamento del niño & 9 & $1,2 \%$ \\
Identidad materna & 8 & $1 \%$ \\
\hline Actitudes respecto a la crianza & 8 & $1 \%$ \\
\hline Flexibilidad & 7 & $0,9 \%$ \\
Autoestima & 7 & $0,9 \%$ \\
Percepción de la experiencia del nacimiento & 5 & $0,6 \%$ \\
\hline Total & 753 & $100 \%$ \\
\hline
\end{tabular}

El primero de estos factores, el apoyo social, se hace explícito a través de un juego propuesto por la psicóloga clínica en relación a la descripción de las dinámicas familiares. A través de él, se desarrolla el rol de la madre, la familia, el padre o compañero, la relación madre-padre y el funcionamiento de la familia; factores sexto, séptimo, noveno y undécimo por mayor orden de aparición.

"El padre y yo estamos juntos, enfrente está mi hija. Separadas del grupo están mi madre y mi suegra, porque a la madre y a la suegra hay que mantenerlas al margen..."

"El niño se sitúa entre el padre y yo. Mi padre se sitúa enfrente. Mi padre es muy niñero y lo malcría, pero en cualquier situación siempre que lo llamo, él viene enseguida. Mi padre siempre me lo ha puesto fácil. Es un incondicional, siempre está ahi”.

Estos cuatro factores se incluyen dentro del microsistema. Se trata de uno de los tres entornos principales que influyen, según la teoría de Mercer, en el proceso de convertirse en madre: el más próximo e íntimo.

"Mi vida social últimamente se reduce a la niña".

"Mañana empiezo a trabajar. Iniciaré el turno de noche. La familia se llevó a la niña al pueblo mientras yo trabajaba. Al principio lo he pasado muy mal".

Continuando con el apoyo social, éste puede clasificarse en emocional, informativo, físico y de valoración. Observándose éstos en las distintas intervenciones que se dan en el taller: 
"Cuando se está con más gente, te relajas y se capta la emoción ambiental porque hay buen clima. Entonces vosotras os relajáis también".

"Lo que me pasa a mi es que estoy muy perdida y necesito de las demás madres, del contacto con otra gente".

Aunque muchas veces el apoyo social pretende proporcionar satisfacción a las madres con la ayuda que ofrecen, éste es vivido por ellas como insatisfactorio:

"Llevo muy mal la presión social que me dice lo que tengo o no tengo que hacer. La enfermera me dijo que cogiera a la niña, la mimara y estuviera con ella. Mi madre me presiona para que no coja a la niña y yo pienso que me queda poco tiempo para empezar a trabajar."

"La opinión de la gente te hace sentir mala madre... Tengo dudas en el dormir en nuestra cama. Mi pareja y yo estamos de acuerdo en ponerlo en la cama y te encuentras en todo momento cuestionada y yo pienso en hacer lo que creo que sea más consecuente..."

También, se observa que a través del apoyo social desarrollado en el grupo, surgen las inquietudes de las madres y el trabajo que éstas realizan en interpretar las señales del lactante, segundo factor en orden de aparición. Esto se pone de manifiesto a través de las respuestas que las madres dan cuando el bebé llora, así como a las demandas de alimentación, descanso y postura, entre otros.

"Enseguida veo en su cara cuando tiene hambre ".

"Tiene calor ahora y por eso llora. Creo que si estuviera desnudo estaría mejor..."

"Se ha descubierto las manos. He observado que acercarse cosas a la cara le relajan y le ayudan a dormirse mejor".

"Le cuesta hacer caca y eso hace que esté más irritable... Tiene cólicos y ya lo he asumido. Él se relaja cuando lo cojo y le hablo".

Si la madre no interpreta correctamente las señales de los lactantes, puede repercutir en su estado emocional, generando tensión debida al rol.

"Es un poco llorona... no sé, a veces me planteo si soy mala madre porque a veces te desespera".

"Cuando el niño llora... no puedo con el llanto del bebé, me tengo que marchar. Entonces necesito de la ayuda de alguien".

A pesar de las tensiones que toda madre puede tener en el desempeño de su rol, ésta siente un vínculo afectivo y emocional hacia su hijo, de aquí surge el factor unión.

"A veces la dejo en el parque, pero no se entretiene mucho, enseguida me llama. Podría estar más entretenida, ¿no? Entonces me voy del parque escondiéndome, pero ella enseguida ve como me alejo y llora. Entonces yo me 
acerco y le voy hablando. La voz le da tranquilidad y con ella le digo que estoy aquí"

"Cuando le pusieron la vacuna lloró un poquito, pero a mí se me saltaban las lágrimas y pensaba ipobre, que tenga que pasar por esto! Qué tontería ¿no? Es una conexión. Es la tensión lo que no puedes evitar que te haga lo que te haga".

La creación de un vínculo entre la madre y su bebé, así como el desarrollo de las tareas de cuidado de rol y el placer y la gratificación que se producen en el desempeño de éste conducen al factor adopción del rol maternal, del que muchas madres manifiestan en ciertos momentos estar en proceso de desarrollo.

"Cada vez conozco mejor a mi bebé. Ya he superado lo de la alimentación...".

"Por la mañana me quedo con ella. Jugamos: ella me mira y sonríe. Yo le pongo la mano y la tranquilizo, entonces le canto y ella me mira. Ella me empieza a sonreír y está más contenta”.

Por último se han identificado otros factores tales como: características del niño, estrés, autoconcepto, identidad maternal, temperamento del niño, gratificaciónsatisfacción, percepción de la experiencia del nacimiento, actitudes respecto a la crianza, autoestima, flexibilidad y estado de salud.

La segunda categoría identificada, "conceptos de Beck" (tabla 3), incluye los factores: ansiedad e inseguridad, estrés en el cuidado del niño, culpabilidad y vergüenza, estrés vital, tristeza materna y labilidad emocional.

Tabla 3: Frecuencia de los ítems relacionados con los conceptos definidos por Beck (3)

\begin{tabular}{lcc}
\hline Ítems conceptos de Beck & Frecuencia absoluta & Frecuencia relativa \\
\hline Inseguridad & 33 & $38,9 \%$ \\
Estrés en el cuidado del niño & 28 & $33 \%$ \\
Culpabilidad y vergüenza & 14 & $16,5 \%$ \\
Estrés vital & 5 & $5,9 \%$ \\
Tristeza materna & 4 & $4,6 \%$ \\
Labilidad emocional & 1 & $1,1 \%$ \\
Total & 85 & $100 \%$ \\
\hline
\end{tabular}

El primer factor, ansiedad e inseguridad, conduce a una sensación de estar sobrepasada en su nuevo papel maternal manifestando una hiperatención en temas relativamente banales.

"Ayer fui a la enfermera a pesarlo porque tenía la sensación de que apenas había cogido peso, pero pesaba bien... La semana que viene lo vacunarán y, no sé, lo paso un poco mal. Antes todo me angustiaba".

"La semana pasada mi hija se tocaba la oreja. Pensé en una infección de oídos y de ahí me vino la idea de una meningitis. La llevé al pediatra de urgencias. La 
conclusión fue que la niña no tenía nada y que realmente lo que se estaba descubriendo es que tenía oídos”.

En el segundo factor, estrés en el cuidado del niño, se evidencian las dificultades expresadas con temas como la alimentación y el descanso.

"Para mi hijo el comer es un momento de irritabilidad. Tengo miedo de que siempre le cueste adaptarse a la comida como ahora"

"Al principio apenas dormía, tenía grietas y problemas con la lactancia materna. Ahora la niña tiene dificultades para comer papillas. Tenía ganas de venir y de ver otras experiencias".

Cabe resaltar que la ansiedad e inseguridad y el estrés en el cuidado del niño pueden repercutir en los sentimientos de culpa-vergüenza que las madres muestran en determinadas situaciones:

"Esta semana he decidido dejar el pecho y darle el biberón. Pero sin darle el pecho tengo remordimientos. Esta noche he dormido seis horas y itengo una alegría en el cuerpo! Pero es que he leído tantas cosas sobre los beneficios de la lactancia materna que... ime sabe mal!"

"Cuando la niña se te pone a llorar, te entran sudores. La gente te dice cosas y ya no sabes qué hacer".

"El inicio del destete ha sido duro porque ella enseguida cuando me la pongo encima se gira para el pecho y eso me desespera, sobre todo por la noche, cuando llora y puede molestar a los vecinos y la niña me pide pecho... y yo le he de dar el biberón"

Por último, comentar que en el transcurso de las sesiones se han podido identificar otros tres factores de Beck. Se trata de estrés vital, tristeza materna y labilidad emocional.

"Es que me entraba una llorera el primer mes que reconozco que estaba insoportable. Tan pronto lloraba como estaba irritable".

"Los primeros momentos pueden ser muy duros. Las emociones personales... yo quería estar contenta pero realmente estaba triste y tenía ganas de llorar..."

Una vez desarrolladas la categoría 1, factores de Mercer, y categoría 2, factores de Beck; se comentará la categoría 3 relacionada con conceptos de promoción de la salud y prevención de la enfermedad.

La categoría 3 (tabla 4), "promoción de la salud y prevención de la enfermedad", incluye cinco ítems. El primero es el desarrollo madurativo constituido por: desarrollo psicomotor y físico, chupete, consejos posturales, hiperestimulación, masaje, actividad acuática, actos esenciales en la crianza, moisés-parque y televisión. 
Tabla 4: Frecuencia de los ítems relacionados con la promoción de la salud y la prevención de la enfermedad.

\begin{tabular}{lcc}
\hline $\begin{array}{l}\text { Categoría 3. Promoción de la salud } \\
\text { y prevención de la enfermedad. }\end{array}$ & $\begin{array}{c}\text { Frecuencia } \\
\text { absoluta }\end{array}$ & $\begin{array}{c}\text { Frecuencia } \\
\text { relativa }\end{array}$ \\
\hline Desarrollo madurativo & 135 & $39,3 \%$ \\
Alimentación & 82 & $24 \%$ \\
Descanso & 56 & $16,3 \%$ \\
Problemas de salud & 48 & $14 \%$ \\
Prevención de accidentes & 22 & $6,4 \%$ \\
Total & 343 & $100 \%$ \\
\hline
\end{tabular}

"Yo veo que mira los muñecos y entonces la pongo en la manta. Ella quiere alcanzar los objetos y llevárselos a la boca pero no atina".

"Quiere estar sentada y se impulsa hacia delante para adoptar la postura. A lo mejor ya la tengo que sentar en el carro".

Los dos siguientes son alimentación y descanso. La alimentación incluiría desde consejos relacionados con la introducción de la alimentación complementaria, lactancia materna, la alimentación con elemento relacional y comunicacional entre padres e hijos, así como los problemas que los padres perciben en la alimentación del bebé.

"Últimamente no coge mucho peso y eso me preocupa. En una semana sólo ha aumentado 80 gramos. No quiere comer. Le doy cada cuatro horas y la niña no me pide..."

"Yo tengo la experiencia de que mi hermana había lactado a mis sobrinos. Pensé que yo lo probaría y que si la experiencia me gustaba lo seguiría haciendo, pero salí del hospital con grietas y una pomada. Yo no disfrutaba de la experiencia. Pero la niña subía de peso. Ahora llevo dos semanas que el pecho no me duele y no tengo grietas. Ahora estoy disfrutando de mi hija".

El descanso se relaciona tanto con consejos sobre los patrones de actividad y descanso normal como con problemas en el sueño, ya sean por hábitos inadecuados o dificultades en mantener un ritmo correcto de vigilia- sueño.

"Se le ha pasado la fiebre y el virus. El problema es que por la noche y por el día ha cambiado el patrón del sueño. Come cada 4 horas. Por el día duerme más tranquila, pero por la noche es un sueño intranquilo. Antes dormía mejor".

"Siempre ha comido y dormido muy bien. Ahora se levanta espantada. Por la noche tiene pesadillas y movimientos rígidos. Eso me preocupa".

El cuarto ítem, manejo problemas de salud (49) está formado por: llanto (20), deposiciones (10), cólicos (5), cuidados de la piel (5), vómitos (3), mucosidad (2), mosquitos (2) y fiebre (1). En este apartado las madres exponen situaciones que les preocupan o han preocupado durante los meses de duración del taller. 
"La semana pasada estuvo cinco días sin hacer caca y yo le hacía masajes porque tiene que cagar sí o sí. El pediatra me dijo que tenía las cacas blandas y que podía tardar, Para mí fue un alivio cuando hizo. Pero, la verdad, es que quieres una solución iya! Por parte del pediatra. Y la gente... unos me decían que le pusiera un bastón con aceite, otros que mirara por internet... pero cuando al fin cagó la niña, era como si cagara yo. Si me llegan a decir la gente que comiera productos amarillos para que mi hija cagara yo me los comía".

"Estos días ha estado muy resfriado. Lo llevamos a urgencias y continúa con mocos y come menos. Se le ve cansado al bebé. Con los mocos lo pasa mal. Yo padecía porque parecía que con los mocos se ahogaba"

Para finalizar, el ítem prevención de accidentes (22) ocuparía el último lugar. Éste se compone de: vacunas (13), accidentes (6), ola de calor (2) e higiene y esterilización (1).

Todos estos factores e ítems surgen a raíz de la dinámica de un taller desarrollado a partir de una propuesta de observación que los profesionales hacen a las madres, encontrando éstas el apoyo de los profesionales y de otras madres en el acompañamiento a esta experiencia, el cual evalúan muy positivamente.

"Me ha gustado ver otras situaciones y cómo otras madres han actuado y a la vez los consejos que nos habéis dado. Estar con otras madres te ayuda a ver las cosas con más naturalidad y como que forman parte de lo que es normal".

"El grupo te permite compartir experiencias. Yo estaba muy angustiada con el pecho. He visto a otras madres que han pasado por lo mismo que yo, las dificultades que se han ido encontrando. He descubierto que soy más paciente".

"Socialmente encuentras mucha presión por hacer las cosas bien. Es como si te evaluaran constantemente. Todo ha sido diferente a como yo creía. Venir aquí me ha ayudado a llevar mejor esa presión. Ya no me pongo tan nerviosa como antes. Las cosas las veo mejor".

\section{DISCUSIÓN}

Entre los resultados más llamativos figuran que las mujeres participantes en este grupo se encuentran, según la teoría de la adopción del rol maternal de Mercer, entre la segunda y cuarta etapa en el proceso de convertirse en madres. El progreso a través de las etapas se verá influenciado por aspectos relacionados con factores de la madre y de su bebé(6,7). Mercer describe un total de veintitrés factores ${ }^{(3,8)}$, algunos de ellos identificados en el apartado resultados correspondientes a la categoría 1.

El que aparece con más frecuencia es el apoyo social, considerando éste como la ayuda que se recibe realmente así como la satisfacción que produce esa ayuda y las personas que la ofrecen ${ }^{(3)}$ pudiendo ser estas personas profesionales y/o o amigos y/o familiares de las madres. El apoyo social debe ser abordado según otros autores (9) debido a que la calidad del mismo resulta factor transcendente en el mantenimiento y agravamiento de la depresión postparto. En las madres participantes, se observa 
que este apoyo se recibe más del microsistema pero aunque éste es el que más aparece, también pueden detectarse el mesosistema y el macrosistema ${ }^{(3,6,8)}$.

A continuación, las señales de los lactantes, es el segundo código que surge más y hace referencia a aquellas conductas que los bebés tienen y que provocan una respuesta a su madre ${ }^{(3)}$.Al respecto, son diversas las ocasiones que las madres manifiestan dificultades en la interpretación de las señales que emiten sus hijos, siendo al mismo tiempo aspecto considerado de relevancia en las intervenciones profesionales según autores ${ }^{(10)}$. Estas situaciones originan estrés en el cuidado del niño, así como sentimientos culpa-vergüenza y/o de ansiedad-inseguridad.

En relación a estos sentimientos, se ha considerado conveniente unir el factor ansiedad de Mercer y ansiedad- inseguridad de Beck. Además, el factor depresión de Mercer se ha sustituido por los factores tristeza materna y labilidad emocional de Beck.

Esta autora indica que los sentimientos de ansiedad-inseguridad, conducen a una sensación de estar sobrepasada en su nuevo papel maternal manifestando una hiperatención en temas relativamente banales ${ }^{(3)}$, conceptos que también refiere Mercer como ansiedad, la cual hace percibir situaciones estresantes como peligrosas o amenazantes.

Por último, describe los sentimientos de culpa-vergüenza como aquellos en los que la mujer percibe que lo está haciendo mal como madre teniendo, en relación a su hijo, pensamientos negativos ${ }^{(3)}$.

En el transcurso de las sesiones se han podido identificar otros tres factores de Beck, de los veintidós que ella identifica en su Teoría de la Depresión Postparto y que complementarían a la teoría de Mercer. Se trata de estrés vital, tristeza materna y labilidad emocional, factores a los que puede haber contribuido el aumento de la sensibilidad materna; aspecto relevante de la atención profesional según algunos autores ${ }^{(10)}$. Se ha optado por estas dos últimas emociones al ser más precisas que la depresión descrita por Mercer: componente afectivo de estar deprimido o tener síntomas depresivos ${ }^{(3)}$.

Los estados emocionales de la madre pueden generar tensión debida al rol, definida por ella como conflictos y dificultades que una madre siente en sus obligaciones en el rol maternal ${ }^{(3)}$.

A pesar de las tensiones que toda madre puede tener en el desempeño de su rol, ésta siente un vínculo afectivo y emocional hacia su hijo ${ }^{(3)}$.La creación de un vínculo entre la madre y su bebé, así como el desarrollo de las tareas de cuidado de rol y el placer y la gratificación que se producen en su desempeño ${ }^{(3)}$ conducen a la adopción del rol maternal ${ }^{(2)}$.

Todos estos factores e ítems surgen a raíz de la dinámica de un taller desarrollado a partir de una propuesta de observación que los profesionales indican a las madres. La observación permite detectar las situaciones que les interesen y quieran comentar. Esto produce una motivación por un aprendizaje que se adapta a las necesidades de cada una de las madres, intentando potenciar en todo momento el saber natural que tienen aun creyendo, ellas mismas, que no lo poseen. Pero limita la uniformidad de los temas a tratar en los talleres que se desarrollan en otras dinámicas grupales. 
A través de la temática desarrollada en este taller, la psicóloga clínica y la enfermera actúan como dos profesionales que se complementan con un objetivo en común: facilitar y fomentar la autonomía por parte de los padres en el cuidado de sus hijos, realizando intervenciones que promuevan la salud y prevengan problemas familiares, en las situaciones de cambios y/o crisis en el ciclo vital de la familia, de esta manera se consigue desarrollar el rol de la familia como promotora de la salud, fortaleciendo la unión y la red familiar ${ }^{(8)}$.

Esto se ha podido observar a partir del análisis de contenidos de este taller, en el que las enfermeras, como profesionales competentes en aspectos conceptuales y técnicos propios de su disciplina, pueden valerse de los modelos de Mercer y Beck para acompañar a las madres en el proceso de convertirse en madres ${ }^{(2)}$.

Pero los profesionales no son los únicos que contestan a las dudas que van surgiendo. El apoyo social que se produce entre las participantes es importante ya que éstas se sienten identificadas con otras personas que se encuentran en su misma situación $n^{(5)}$.

Eso hace que la participación de las madres en una dinámica grupal como la descrita puede sea de utilidad al proporcionar apoyo social, influencia en las percepciones de sus capacidades sobre la maternidad, disminuyendo el estrés y la ansiedad y mejorando el afrontamiento de los padres así como el funcionamiento efectivo de la familia $^{(11,12)}$.

El apoyo social que se produce entre las participantes es importante ya que éstas se sienten identificadas con otras personas que se encuentran en su misma situación ${ }^{(5)}$. Esto pone de manifiesto la necesidad del diálogo y de la escucha empática para comprender las preocupaciones y ganar confianza en las capacidades de las madres como cuidadoras ${ }^{(6)}$.

Para finalizar, a pesar de que la evidencia es limitada sobre la manera de fomentar los sentimientos de la madre acerca de sí misma y el apego a su bebé(7), y de que las intervenciones grupales relacionadas con el cuidado del niño sano no tienen ningún efecto en el sentido de la competencia maternal, la percepción social, el apoyo o el aislamiento, la autoestima, la ansiedad o la depresión en las madres de alto riesgo ${ }^{(12)}$; las madres manifiestan una evaluación positiva en la participación de los talleres.

No obstante, sería interesante desarrollar investigaciones que permitan analizar los contenidos de este tipo de talleres utilizando modelos conceptuales propios de la disciplina enfermera así como considerar una evaluación cualitativa de las madres participantes utilizando diferentes instrumentos propios de esta metodología.

\section{CONCLUSIONES}

Este estudio ha descrito y relacionado los contenidos de las dinámicas grupales con el desarrollo del rol maternal identificando 3 categorías y 50 códigos. Para ello ha empleado una metodología cualitativa, con enfoque etnográfico, la cual ha utilizado la observación participante y el diario de campo.

Los modelos de Mercer y Beck han sido de utilidad para valorar, planificar, ejecutar y evaluar el cuidado enfermero a las madres y sus bebés en sus intervenciones tanto de manera autónoma con en colaboración con otros profesionales. 


\section{Agradecimientos}

Se reconoce al Colegio de Enfermería de Barcelona que ha aportado su ayuda para el desarrollo de esta investigación concediendo a este proyecto una financiación parcial en el marco de ayudas a Proyectos de Investigación PR 5326-11

\section{REFERENCIAS}

1. Stern, DN. El nacimeinto de una madre. Barcelona: Paidós, 2006.

2. Mercer, RT. Becoming a Mother. Journal of Nursing Scholarship. Third Quarter 2004. 36 (3): 226-232.

3. Marriner, A; Raile, M. Modelos y teorías en enfermería. Barcelona: Elsevier Mosby, 2011 ( $7^{\mathrm{a}}$ ed.).

4. Lima Rodríguez, Joaquín Salvador; Cobo Castillo, Celia; Lima Serrano, Marta; Casado Mejía, Rosa. Educación para la Salud Grupal: ¿Es utilizada por las enfermeras de Atención Primaria de Salud? Enfermería Comunitaria (rev. digital) 2008, 4(2). Disponible en <http://www.indexf.com/comunitaria/v4n2/ec6688.php> Consultado el 27 de Junio de 2011.

5. Nelson, AM. Transition to Motherhood. JOGNN. 2003; 32 (4): 465-477.

6. Mercer, RT. Nursing Support of the Process of Becoming a Mother. JOGNN. 2006. 35 (5): $649-651$

7. Orden SAS/1729/2010, de 17 de junio, por la que se aprueba y publica el programa formativo de la especialidad de Enfermería Familiar y comunitaria

8. Mercer, RT; Walker, LO. A Review of Nursing Interventions to Foster Becoming a Mother. JOGN. 2006; 35 (5): 568-581.

9. Tedder JL. Give Them The HUG: An Innovative Approach to Helping Parents Understand the Language of Their Newborn. J Perinat Educ. 2008 Spring;17(2):14-20.

10. Gómez SL. Representaciones maternales en madres primerizas que presentan sintomatología depresiva postparto [Tesis Doctoral]. Lima. Perú: Pontificia Universidad católica del Perú; 2011.

11.Gardner, MR; Deatrick, JA. Understanding interventions and outcomes in mothers of infants. Issues in Comprehensive Pediatric Nursing. 2006; 29: 25-44.

12. Kruske S, Schimied V, Sutton F, O'Hare J. Mother's experiencies of Facilitated Peer support groups and Individual Child health Nursing Support: A comparative Evaluation. The Journal of Perinatal Education. 2004; 13 (3): 31-38. 